\title{
Optimal Control Problems Arising in the Zinc Sulphate Electrolyte Purification Process
}

\author{
Ling Yun Wang ${ }^{1,2,3}$, Wei Hua Gui ${ }^{1}$, Kok Lay Teo ${ }^{2 *}$ Ryan Loxton ${ }^{2}$, Chun Hua Yang ${ }^{1}$ \\ 1. School of Information Science and Engineering, \\ Central South University, Changsha, China, 410083 \\ 2. Department of Mathematics and Statistics, \\ Curtin University of Technology, Perth, Australia, 6105 \\ 3. School of Electrical Engineering and Renewable Energy, \\ China Three Gorges University, Yichang, China, 443002
}

\begin{abstract}
Before zinc electrolysis, zinc powder is added to the zinc sulphate electrolyte solution to facilitate the removal of harmful metallic ions. This purification process can be modeled by a time delay differential equation. Since some of the parameters in this model are unknown, zinc powder is normally added excessively. We use an optimization technique to estimate the unknown parameters from experimental data. Then, we formulate an optimal control problem to minimize the amount of zinc powder added. We solve this optimal control problem numerically by using the control parametrization method. The results indicate that the amount of zinc powder added can be decreased, on average, by approximately $7 \%$.
\end{abstract}

Keywords: Zinc sulphate electrolyte; Purification process; Continuous stirred tank reactor; Optimal control; Time delayed system; Multiple characteristic time points.

\section{Introduction}

The zinc industry is one of the most important nonferrous industries in China. Demand for zinc is increasing annually because of the Chinese economy's rapid development. Most zinc smelting production processes, which include roasting, leaching, purification

\footnotetext{
*Corresponding author: k.l.teo@curtin.edu.au
} 
and electrolysis, are hydrometallurgical. In zinc electrolysis, the purity of the zinc sulphate solution is imperative. In the raw zinc sulphate solution, obtained from a neutral thickener used for separating zinc sulphate solution from leached residue in the leaching process, most of the impurities are metallic ions in their sulphate form. These impurities, which include cobalt and cadmium ions, are harmful for zinc electrolysis. Even a small amount of these metallic ions in the solution may hinder zinc deposition during the electrolysis process. Existing research has focussed on the effect of impurities on zinc electrolysis. For example, Bratt [1] stated the effect of impurities by estimating the hydrogen overpotential, which influences the zinc electrolysis significantly, using a mathematical equation. Also, Fosnacht and O'Keefe [2] designed experiments to quantify the interactions of impurity ions.

The primary objective of the zinc sulphate solution purification process is to obtain an electrolyte solution that is suitable for zinc electrolysis. Since zinc powder is expensive, its consumption should be minimized during the purification process. This has been the subject of many papers, see [3-5]. Most research (see, [6-12]) has focused on experiments for determining the optimum purification conditions, such as solution temperature, zinc dust particle size, the quantity of zinc dust added, activators concentration and reaction time. In addition, some works (see, for example, [15]) have discussed the modeling and control of the zinc sulphate solution purification process in a special scenario.

The control parametrization technique, proposed by Teo, Goh and Wong (see, [24]) has been applied to a large variety of practical problems. We will use this optimal control method to solve the problem of minimizing zinc powder consumption in the zinc sulphate electrolyte purification process.

First, based on the deposition reaction mechanism and chemical kinetics, we establish a dynamical model for the purification process of removing cobalt and cadmium ions - the two main metallic ion impurities when zinc powder is present in the reaction tank. Then, the unknown model parameters are identified using a gradient-based optimization technique. After this, the problem of minimizing the zinc powder consumption is formulated as a time delayed optimal control problem subject to continuous state inequality constraints. By approximating the control as a piecewise constant function, this optimal control problem is reduced to a nonlinear optimization problem that can also be solved using gradient-based optimization method.

\section{System Description}

In this section, we will describe the mathematical model for the second stage of the zinc sulphate purification process.

In a typical zinc production factory in China, the purification system consists of three stages. In the first stage, the zinc sulphate solution obtained from the leaching process passes through two agitated reaction tanks. Zinc powder is added, whose 
quantity is controlled by an electronic apparatus, at the inlet of the reaction tanks to facilitate the deposition of cadmium, cobalt, copper and nickel ions. During this stage, the cobalt and cadmium ion concentrations are reduced to a relatively stable level. The concentrations of the other metallic ion impurities can also be reduced to acceptable levels.

After filtration and reheating, the electrolyte solution is supplied to the second stage, where a combination of zinc powder and antimony tartrate with appropriate amount is added at the inlet of the second stage. This stage removes the cobalt ions at higher solution temperature $85-90^{\circ} \mathrm{C}$, meanwhile cadmium ions are also deposited continually in this stage. In fact, zinc sulphate solution purification often involves considerable difficulties. The deposition of cobalt ions with zinc powder is a relatively slow process, but it may be accelerated by adding small quantities of catalyst, such as antimony tartrate, at higher solution temperature. After the second stage, the cobalt and cadmium ion concentrations can be reduced to acceptable levels. Finally, the solution is fed to the third stage, where a small quantity of zinc powder is added to deposit the cadmium ions, which is redissolved into the solution again. After three stages, the concentrations of the metallic ion impurities in the zinc sulphate electrolyte solution are reduced to acceptable levels. Then the purified zinc sulphate solution is fed to the electrolysis tanks after filtration and cooling.

Generally, the grade of the zinc ore for smelting is low and its composition is complex. Also there are many kinds of impurities. Usually, the zinc powder is added liberally in the first stage to guarantee that the concentrations of the metallic ion impurities are decreased to relatively low levels. This ensures that the subsequent purification stage runs normally. Thus, the amount of zinc powder added in the first stage is always the maximum value. The most critical stage of the entire purification process is the second stage, which is the removal of cobalt and cadmium ions by deposition with zinc powder. As mentioned above, the cobalt and cadmium ion concentrations can be reduced to relatively low values after the first stage. Hence, the addition of zinc powder can be regulated according to the measured cobalt and cadmium ion concentrations at the inlet of the reaction tank in the second stage. In the third stage of the purification process, a much smaller quantity of zinc powder is added to the reaction tanks to remove the redissolved cadmium ions. Therefore, our research will focus on using chemical reaction laws to establish a mathematical model of the second stage purification process, and then apply optimal control techniques to determine the optimal rate of zinc powder addition.

\subsection{Deposition reaction mechanism}

Before establishing the purification process model, we will describe the deposition reaction mechanism of the noble metallic ions in the solution. In most noble metallic ion deposition reaction processes, deposition has been found to be controlled by boundary layer diffusion, and the reaction is first-order with respect to the deposition of noble 
metallic ions. The generally accepted differential equation for describing the kinetics of the deposition process has been derived under the following assumptions:

(1) The metal powder particles are spherical;

(2) The diffusion of metallic ions in the solid particles is described by Fick's law of diffusion;

(3) The diffusion process is in the radial direction only;

(4) The replacement reaction takes place in isothermal conditions.

Under these assumptions, if we take into consideration of the fact that the metallic ion concentration decreases continuously with time, and the reaction surface area of the metal powder particles does not change during the reaction, then the following differential equation can be derived (see $[8,9]$ ).

$$
\frac{\mathrm{d} C}{\mathrm{~d} t}=-\frac{D U}{\rho V} C=-\frac{k U}{V} C,
$$

where $C$ is the metallic ion concentration, $t$ is the reaction time, $\mathrm{d} C / \mathrm{d} t$ is the deposition reaction rate, $D$ is the diffusion coefficient, $\rho$ is the diffusion layer thickness, $k$ is the reaction rate coefficient, $U$ is the reaction surface area of the solid particles, and $V$ is the volume of the solution. Generally, equation (2.1) reflects the relationship between the metallic ion concentration and the reaction surface area of solid particles in the solution.

Usually the reaction rate coefficient is determined by the different solution conditions such as reactant concentration and temperature. Some experiments (see [10-12]) were designed to obtain the reaction rate coefficient. These experiments are carried out under the particular conditions. However, there is no general experimental method for directly calculating the reaction rate coefficient of the metallic ion deposition process in the flowing solution.

\subsection{Mathematical model}

In the case of the practical industrial process, the zinc powder is added continuously to the zinc sulphate solution at the inlet of the purification reaction tank to remove the metallic ion impurities. Generally, only the cobalt and cadmium ions concentrations at the inlet and outlet of the reaction tank are respectively measured at each hour. The addition of zinc powder is adjusted at each hour according to the cobalt and cadmium ions concentrations. Meanwhile, it usually takes two hours for zinc sulphate solution to flow from the inlet to the outlet of the reaction tank in the second stage. That is, the reaction time for the second stage is two hours. This is a time delayed reaction process. But considering the complicated reaction environment and uncertainties, the 
zinc powder is added much more excessively than required so as to guarantee the quality of the production. Thus, a large amount of zinc powder is wasted. Clearly, this is not desirable in practice.

In fact, there are other metallic ion impurities in the zinc sulphate solution. However, they will decrease if the cobalt and cadmium ions concentrations are reduced in the zinc sulphate electrolyte solution. Here, we assume that the zinc powder only involves in zinc-cobalt and zinc-cadmium deposition reaction. Thus, there are two main replacement reactions between the metallic ion impurities and the zinc powder particles occurring in the zinc sulphate electrolyte solution. The reactions, which are relevant to zinc-cobalt and zinc-cadmium deposition reaction, are described by the following chemical reaction equations (see $[3-5,9]$ ):

$$
\begin{aligned}
& \mathrm{Co}^{2+}+\mathrm{Zn} \rightarrow \mathrm{Zn}^{2+}+\mathrm{Co} \downarrow \\
& \mathrm{Cd}^{2+}+\mathrm{Zn} \rightarrow \mathrm{Zn}^{2+}+\mathrm{Cd} \downarrow
\end{aligned}
$$

It is assumed that these reactions will be maintained continuously as long as there are zinc powder particles with sufficiently small size in the solution. Meanwhile, there is also likely some coupling reactions between different metallic ions (see [14]). For example, the replacement reaction between cobalt ions and zinc powder particles will be influenced by the cadmium ions or the other metallic ion impurities. To describe the dynamical behavior of the metallic ions deposition reaction process, component balance equations must be developed. This reaction process can be considered to occur in the ideal continuous stirred tank reactor (CSTR), moreover, the coupling items and time delay are also incorporated in this model. Much work has been done on the research about CSTR, see, for example [15-17]. In recent years, a variety of approaches have been used in the study of the synthesis of estimation and control algorithm for CSTR in the chemical engineering. Applications of modern estimation and control technique to CSTR have been extensively reported in the last few years. For example, Lynch and Ramirez [18] designed a time optimal controller with a Kalman filter for state estimation in CSTR; Knapp, Budman and Broderick [19] used an adaptive control with a neural network model approach in CSTR; Cebuhar and Costanza [20] applied a bilinear optimal control method to CSTR. However, few papers have applied the knowledge of CSTR to the reaction between metallic ions and solid particles. The second stage purification process can be described as the following dynamical equations:

$$
\begin{aligned}
& V \frac{\mathrm{d} C_{1}(t)}{\mathrm{d} t}=Q C_{10}-Q C_{1}(t-2)-k_{1} U_{1} C_{1}(t-2)+\alpha C_{2}(t-2), \\
& V \frac{\mathrm{d} C_{2}(t)}{\mathrm{d} t}=Q C_{20}-Q C_{2}(t-2)-k_{2} U_{2} C_{2}(t-2)+\beta C_{1}(t-2) .
\end{aligned}
$$

These equations are given under the above assumptions. We can see that these chemical reaction equations are similar because the reaction properties of zinc-cobalt 
and zinc-cadmium are similar. In the above equations, $C_{1}(t)$ and $C_{2}(t)$ represent cobalt and cadmium ion concentrations in the reaction tank respectively; $C_{10}$ and $C_{20}$ represent cobalt and cadmium ion concentrations at the entry of the reaction tank; $Q$ is the flux of solution; $V$ is the volume of the reaction tank; $k_{1}$ and $k_{2}$ are reaction rate coefficients for cobalt and cadmium ion, respectively; $\alpha$ and $\beta$ are coupling coefficients; $U_{1}$ and $U_{2}$ are zinc powder particles reaction surface areas for the two metallic ions. They are control variables and are converted from the weight of zinc powder because the relationship between reaction surface area and weight is linear through the experiment validation. In this group of equations, $C_{10}, C_{20}, Q$ and $V$ are known parameters for computation in a period of time. We shall first use the optimal control theory to be described in the next section to find the values of these unknown parameters $k_{1}, k_{2}, \alpha$ and $\beta$, which best fit the observed data. In this exercise, the amount of $U_{1}$ and $U_{2}$ are the actual amounts that are used, though much too excessive than required. Also, the values of $C_{1}$ and $C_{2}$ for time prior to zero are obtained through interpolation of the measured data by using cubic basis spline interpolation method (see, [25]).

By using the values of these parameters so obtained in the equations (2.2), we formulate an optimal control problem, where the controls $U_{1}$ and $U_{2}$ are minimized subject to the condition that the deviation of the corresponding values of $C_{1}(t)$ and $C_{2}(t)$ from the previously obtained ones are within acceptable allowance at each time point. The solution method is in the next section.

To close this section, we wish to remark that the amount of zinc powder is evaluated as the weight, rather than the reaction surface area, in the practical production process. However, suppose that the zinc powder particles are spherical, taking into account the physical characteristics of the zinc powder, the relationship between the reaction surface area and weight $G$ of zinc powder is $U=1740\left(\mathrm{~m}^{2} / \mathrm{kg}\right) \times G(\mathrm{~kg})$. The coefficient in this formula is measured by using a BET analyzer which is described in [10]. Clearly, the reaction surface area obtained from the dynamical equations could be easily converted to the weight of the zinc powder. This is then used in operation.

\section{Gradient-based optimization method}

Control parametrization is a computational method for solving general optimal control problems. This technique generates a sequence of approximation to the control using piecewise constant functions with pre-assigned switching points. In this process, the heights of the piecewise constant functions are regarded as decision variables which are control parameters. Each of the approximate problems is solved as a nonlinear programming problem by using a gradient-based strategy. The explanation of this theory is given in [24].

Consider a process described by the following system of time delayed differential 
equations defined on the fixed time interval $(0, T]$ :

$$
\frac{\mathrm{d} \boldsymbol{x}(t)}{\mathrm{d} t}=\boldsymbol{f}(t, \boldsymbol{x}(t), \boldsymbol{x}(t-h), \boldsymbol{u}(t), \boldsymbol{\zeta}),
$$

where $\boldsymbol{x}=\left[x_{1}, \ldots, x_{n}\right]^{\top} \in \mathbb{R}^{n}, \boldsymbol{u}=\left[u_{1}, \ldots, u_{r}\right]^{\top} \in \mathbb{R}^{r}, \boldsymbol{\zeta}=\left[\zeta_{1}, \ldots, \zeta_{s}\right]^{\top} \in \mathbb{R}^{s}$ are, respectively, the state, control and system parameter vectors; $\boldsymbol{f}=\left[f_{1}, \ldots, f_{n}\right]^{\top} \in \mathbb{R}^{n}$; $h$ is the time delay satisfying $0<h<T$. The initial function for the differential equation (3.1) is

$$
\begin{gathered}
\boldsymbol{x}(t)=\boldsymbol{\phi}(t), \quad t \in[-h, 0), \\
\boldsymbol{x}(0)=\boldsymbol{x}^{0},
\end{gathered}
$$

where $\phi(t)=\left[\phi_{1}(t), \ldots, \phi_{n}(t)\right]^{\top}$ is a given continuous function from $[-h, 0)$ into $\mathbb{R}^{n}$, and $\boldsymbol{x}^{0}$ is a given vector in $\mathbb{R}^{n}$.

The system of dynamical equations (2.2) is clearly a special case of system (3.1) with initial conditions (3.2). We shall present computational methods for solving two classes of time delayed optimal control problems involving system (3.1) with initial conditions (3.2). They are then applied to the study of the zinc sulphate electrolyte purification process involving the system (2.2).

Let $t_{i}, i=1, \ldots, N$, be given time points in $[0, T]$. We assume that the control takes the form given below:

$$
\begin{gathered}
\boldsymbol{u}^{p}(t)=\gamma(t), \quad t \in[-h, 0), \\
\boldsymbol{u}^{p}(t)=\sum_{i=1}^{N_{p}} \boldsymbol{\sigma}^{p, i} \chi_{\left[t_{i-1}, t_{i}\right)}(t), \quad t \in[0, T],
\end{gathered}
$$

where $\gamma(t)=\left[\gamma_{1}(t), \ldots, \gamma_{r}(t)\right]^{\top}$ is a given piecewise continuous function from $[-h, 0)$ into $\mathbb{R}^{r}$, while $\chi_{\left[t_{i-1}, t_{i}\right)}$ denotes the indicator function of the interval $\left[t_{i-1}, t_{i}\right)$ defined by

$$
\chi_{I}(t)= \begin{cases}1, & \text { if } t \in I \\ 0, & \text { otherwise. }\end{cases}
$$

Define $\boldsymbol{\sigma}^{p, i}=\left[\sigma_{1}^{p, i}, \ldots, \sigma_{r}^{p, i}\right]^{\top}$ and $\boldsymbol{\sigma}^{p}=\left[\left(\boldsymbol{\sigma}^{p, 1}\right)^{\top}, \ldots,\left(\boldsymbol{\sigma}^{p, N_{p}}\right)^{\top}\right]^{\top}$. Let $U \subset \mathbb{R}^{r}$ be both compact and convex. A function $\boldsymbol{u}$ given by (3.3b) with $\boldsymbol{\sigma}^{p, i} \in U, i=1, \ldots, N_{p}$, is called an admissible control. Let $\mathcal{U}^{p}$ be the set of all such admissible controls, and let $\Theta^{p}$ be the set containing all $\boldsymbol{\sigma}^{p}=\left[\left(\boldsymbol{\sigma}^{p, 1}\right)^{\top}, \ldots,\left(\boldsymbol{\sigma}^{p, N_{p}}\right)^{\top}\right]^{\top}$ with $\boldsymbol{\sigma}^{p, i} \in U, i=1, \ldots, N_{p}$. Clearly, each $\boldsymbol{u} \in \mathcal{U}^{p}$ corresponds uniquely to a $\boldsymbol{\sigma}^{p} \in \Theta^{p}$ and vice versa. Let $Z \subset \mathbb{R}^{s}$ be both compact and convex, and we assume that $\boldsymbol{\zeta}=\left[\zeta_{1}, \ldots, \zeta_{s}\right]^{\top} \in Z$. With $\boldsymbol{u} \in \mathcal{U}^{p}$, the system of time delayed differential equations (3.1) can be written as:

$$
\frac{\mathrm{d} \boldsymbol{x}(t)}{\mathrm{d} t}=\tilde{\boldsymbol{f}}\left(t, \boldsymbol{x}(t), \boldsymbol{x}(t-h), \boldsymbol{\sigma}^{p}, \boldsymbol{\zeta}\right),
$$


where

$$
\tilde{\boldsymbol{f}}\left(t, \boldsymbol{x}(t), \boldsymbol{x}(t-h), \boldsymbol{\sigma}^{p}, \boldsymbol{\zeta}\right)=\boldsymbol{f}\left(t, \boldsymbol{x}(t), \boldsymbol{x}(t-h), \sum_{i=1}^{N_{p}} \boldsymbol{\sigma}^{p, i} \chi_{\left[t_{i-1}, t_{i}\right)}(t), \boldsymbol{\zeta}\right) .
$$

We may now state a time delayed optimal control problem with multiple characteristic time points appearing in the cost and constraint functions as follows:

Problem (P1). Given the dynamical system (3.4) with initial conditions (3.2), find a parameter vector $\left(\boldsymbol{\sigma}^{p}, \boldsymbol{\zeta}\right) \in \Theta^{p} \times Z$ such that the cost function

$$
g_{0}\left(\boldsymbol{\sigma}^{p}, \boldsymbol{\zeta}\right)=\Phi_{0}\left(\boldsymbol{x}\left(\tau_{1} \mid \boldsymbol{\sigma}^{p}, \boldsymbol{\zeta}\right), \ldots, \boldsymbol{x}\left(\tau_{M+1} \mid \boldsymbol{\sigma}^{p}, \boldsymbol{\zeta}\right)\right)+\int_{0}^{T} \tilde{\mathcal{L}}_{0}\left(t, \boldsymbol{x}(t), \boldsymbol{x}(t-h), \boldsymbol{\sigma}^{p}, \boldsymbol{\zeta}\right) \mathrm{d} t
$$

is minimized subject to the following canonical inequality constraints:

$$
\begin{aligned}
g_{m}\left(\boldsymbol{\sigma}^{p}, \boldsymbol{\zeta}\right)= & \Phi_{m}\left(\boldsymbol{x}\left(\tau_{1} \mid \boldsymbol{\sigma}^{p}, \boldsymbol{\zeta}\right), \ldots, \boldsymbol{x}\left(\tau_{M+1} \mid \boldsymbol{\sigma}^{p}, \boldsymbol{\zeta}\right)\right) \\
& +\int_{0}^{T} \tilde{\mathcal{L}}_{m}\left(t, \boldsymbol{x}(t), \boldsymbol{x}(t-h), \boldsymbol{\sigma}^{p}, \boldsymbol{\zeta}\right) \mathrm{d} t \geq 0, \quad m=1, \ldots, N,
\end{aligned}
$$

where the time points $\tau_{i}, 0<\tau_{i}<T, i=1, \ldots, M$, are referred to as the characteristic time points, while

$\tilde{\mathcal{L}}_{m}\left(t, \boldsymbol{x}(t), \boldsymbol{x}(t-h), \boldsymbol{\sigma}^{p}, \boldsymbol{\zeta}\right)=\mathcal{L}_{m}\left(t, \boldsymbol{x}(t), \boldsymbol{x}(t-h), \sum_{i=1}^{N_{p}} \boldsymbol{\sigma}^{p, i} \chi_{\left[t_{i-1}, t_{i}\right)}(t), \boldsymbol{\zeta}\right), \quad m=0,1, \ldots, N$

We use the conventions that $\tau_{0}=0$ and $\tau_{M+1}=T$.

In practice, many constraints do not appear as in the form of canonical constraints. Rather, they appear in the form of continuous state inequality constraints. We may now describe the corresponding optimal control problem as follows:

Problem (P2). Given the dynamical system (3.4) with initial conditions (3.2), find a $\left(\boldsymbol{\sigma}^{p}, \boldsymbol{\zeta}\right) \in \Theta^{p} \times Z$ such that the cost function

$$
g_{0}\left(\boldsymbol{\sigma}^{p}, \boldsymbol{\zeta}\right)=\Phi_{0}\left(\boldsymbol{x}\left(T \mid \boldsymbol{\sigma}^{p}, \boldsymbol{\zeta}\right)\right)+\int_{0}^{T} \tilde{\mathcal{L}}_{0}\left(t, \boldsymbol{x}(t), \boldsymbol{x}(t-h), \boldsymbol{\sigma}^{p}, \boldsymbol{\zeta}\right) \mathrm{d} t
$$

is minimized over $\Theta^{p} \times Z$ subject to the continuous state inequality constraints defined by

$$
h_{i}\left(t, \boldsymbol{x}\left(t \mid \boldsymbol{\sigma}^{p}, \boldsymbol{\zeta}\right), \boldsymbol{\zeta}\right) \geq 0, \quad t \in[0, T], \quad i=1, \ldots, N,
$$

where $h_{i}, i=1, \ldots, N$, are continuously differentiable functions defined on $[0, T] \times$ $\mathbb{R}^{n} \times \mathbb{R}^{s}$.

Using the constraint transcription method introduced in Chapter 8 of [24], each of these continuous state inequality constraints is approximated by the inequality con- 
straint in canonical form

$$
\gamma+\int_{0}^{T} \mathcal{L}_{i, \varepsilon}\left(t, \boldsymbol{x}\left(t \mid \boldsymbol{\sigma}^{p}, \boldsymbol{\zeta}\right), \boldsymbol{\zeta}\right) \mathrm{d} t \geq 0
$$

where

$$
\mathcal{L}_{i, \varepsilon}\left(t, \boldsymbol{x}\left(t \mid \boldsymbol{\sigma}^{p}, \boldsymbol{\zeta}\right), \boldsymbol{\zeta}\right)= \begin{cases}h_{i}, & \text { if } h_{i}<-\varepsilon \\ -\left(h_{i}-\varepsilon\right)^{2} / 4 \varepsilon, & \text { if }-\varepsilon \leq h_{i} \leq \varepsilon \\ 0, & \text { if } h_{i}>\varepsilon\end{cases}
$$

$\varepsilon>0$ is a smoothing parameter, which is a small adjustable quantity. It is shown in the reference [24] that, under appropriate assumption, there exists a $\gamma(\varepsilon)>0$ such that for each $\gamma, 0<\gamma<\gamma(\varepsilon)$, if an admissible $\left(\boldsymbol{\sigma}^{p}, \boldsymbol{\zeta}\right) \in \Theta^{p} \times Z$ satisfies the constraints (3.9), it also satisfies the constraints (3.8). Thus, optimal control problems involving continuous state inequality constraints of the form (3.8) can always be approximated by a sequence of optimal control problems involving inequality constraints in canonical form, i.e. in the form of Problem (P1).

To solve Problem (P1) as a mathematical programming problem by using a gradientbased technique, such as the sequential quadratic programming (SQP) approximation scheme, it is required to know, for a given $\left(\boldsymbol{\sigma}^{p}, \boldsymbol{\zeta}\right) \in \Theta^{p} \times Z$, the values of the cost function (3.5) and the constraint functions (3.9). We also require the information of their gradients. The calculation of the values of the cost function (3.5) and the constraint functions (3.9) can be achieved quite easily. We solve, for a given $\left(\boldsymbol{\sigma}^{p}, \boldsymbol{\zeta}\right) \in \Theta^{p} \times Z$, the corresponding solution of the system (3.4) with initial condition (3.2). Then, the values of the cost and constraint functions can be calculated readily. To calculate the gradients of these functions, we have the following theorem, which presents the required gradient formulae.

Theorem 1. Consider Problem (P1). For each $m=0,1, \ldots, N$, the gradients of the function $g_{m}$ with respect to $\boldsymbol{\sigma}^{p}$ and $\boldsymbol{\zeta}$ are given by

$$
\begin{aligned}
\frac{\partial g_{m}\left(\boldsymbol{\sigma}^{p}, \boldsymbol{\zeta}\right)}{\partial \boldsymbol{\sigma}^{p, i}} & =\sum_{i=1}^{M+1} \int_{\tau_{i-1}}^{\tau_{i}} \frac{\partial H_{m}}{\partial \boldsymbol{\sigma}^{p, i}} \chi_{\left[\tau_{i-1}, \tau_{i}\right)} \mathrm{d} t \\
\frac{\partial g_{m}\left(\boldsymbol{\sigma}^{p}, \boldsymbol{\zeta}\right)}{\partial \boldsymbol{\zeta}} & =\sum_{i=1}^{M+1} \int_{\tau_{i-1}}^{\tau_{i}} \frac{\partial H_{m}}{\partial \boldsymbol{\zeta}} \mathrm{d} t
\end{aligned}
$$

where

$$
H_{m}=\tilde{\mathcal{L}}_{m}\left(t, \boldsymbol{x}(t), \boldsymbol{x}(t-h), \boldsymbol{\sigma}^{p}, \boldsymbol{\zeta}\right)+\left(\boldsymbol{\lambda}^{\boldsymbol{m}}\right)^{\top} \tilde{\boldsymbol{f}}\left(t, \boldsymbol{x}(t), \boldsymbol{x}(t-h), \boldsymbol{\sigma}^{p}, \boldsymbol{\zeta}\right),
$$

and $\boldsymbol{\lambda}^{m}(t)$ is the corresponding solution of the respective co-state system defined by

$$
\frac{\mathrm{d}\left(\boldsymbol{\lambda}^{m}(t)\right)^{\top}}{\mathrm{d} t}=-\frac{\partial H_{m}}{\partial \boldsymbol{x}}-\frac{\partial \hat{H}_{m}}{\partial \boldsymbol{x}},
$$


where $t \in\left(\tau_{i-1}, \tau_{i}\right), i=1, \ldots, M+1$, with the jump conditions

$$
\boldsymbol{\lambda}^{m}\left(\tau_{i}^{+}\right)^{\top}-\boldsymbol{\lambda}^{m}\left(\tau_{i}^{-}\right)^{\top}=-\frac{\partial \Phi_{m}\left(\boldsymbol{x}\left(\tau_{1}\right), \ldots, \boldsymbol{x}\left(\tau_{M+1}\right)\right)}{\partial \boldsymbol{x}\left(\tau_{i}\right)}, \text { for } \quad i=1, \ldots, M,
$$

and the terminal condition

$$
\begin{gathered}
\left(\boldsymbol{\lambda}^{m}(T)\right)^{\top}=\frac{\partial \Phi_{m}\left(\boldsymbol{x}\left(\tau_{1}\right), \ldots, \boldsymbol{x}\left(\tau_{M}\right), \boldsymbol{x}(T)\right)}{\partial \boldsymbol{x}(T)} \\
\boldsymbol{\lambda}^{m}(t)=0, \quad t>T
\end{gathered}
$$

where

$$
\begin{gathered}
\hat{\boldsymbol{\lambda}}^{m}(t)=\boldsymbol{\lambda}^{m}(t+h), \\
\hat{H}_{m}=\tilde{\mathcal{L}}_{m}\left(t+h, \boldsymbol{x}(t+h), \boldsymbol{x}(t), \boldsymbol{\sigma}^{p}, \boldsymbol{\zeta}\right) e(T-t-h) \\
+\left(\hat{\boldsymbol{\lambda}}^{m}\right)^{\top} \tilde{\boldsymbol{f}}\left(t+h, \boldsymbol{x}(t+h), \boldsymbol{x}(t), \boldsymbol{\sigma}^{p}, \boldsymbol{\zeta}\right) e(T-t-h),
\end{gathered}
$$

and $e(\cdot)$ is the unit step function.

Proof. The proof follows the steps of the proof given for Theorem 5.5.1 in Chapter 5 of [24] but with the terms involving the multiple characteristic time points being handled by the approach given for the proof of Theorem 4.3 in Chapter 4 of [21].

From Theorem 1, we see that we can calculate, for each $\left(\boldsymbol{\sigma}^{p}, \boldsymbol{\zeta}\right) \in \Theta^{p} \times Z$, the gradients of the cost and constraint functions. Hence, Problem (P1) can be solved as a nonlinear optimization problem by using a gradient-based technique. Here, the SQP approximation scheme is used.

\section{Simulation}

We now return to the problem of optimal control of the zinc sulphate electrolyte solution purification process. Consider the dynamical model given in Section 2.2. Let $\hat{C}_{1}(i)$ and $\hat{C}_{2}(i)$ denote, respectively, the observed data of concentrations of cobalt and cadmium ions at the measurement time points $i=1, \ldots, 8$. Our first aim is to choose the optimal system parameters $k_{1}, k_{2}, \alpha, \beta$ and the control $\left(U_{1}^{p}, U_{2}^{p}\right)$, where $U_{1}^{p}$ and $U_{2}^{p}$ are parameterized by (3.3b), i.e.

$$
\begin{aligned}
& U_{1}^{p}(t)=\sum_{i=1}^{8} \sigma_{1}^{p, i} \chi_{[i-1, i)}(t), \\
& U_{2}^{p}(t)=\sum_{i=1}^{8} \sigma_{2}^{p, i} \chi_{[i-1, i)}(t),
\end{aligned}
$$


so that the trajectory "best" fits the observed data at these measurement time points. However, the total quantity of added zinc powder is known, so $\Gamma$ is taken as a total zinc powder reaction surface area. Here, we set $U_{2}^{p}=\Gamma-U_{1}^{p}$. Thus, only $U_{1}^{p}$ is a control to be obtained. We now need to find four parameters $k_{1}, k_{2}, \alpha, \beta$ and a control $U_{1}^{p}$, being parameterized by (4.1a), such that the following cost function

$$
J_{1}=\sum_{i=1}^{8}\left[\left(C_{1}(i)-\hat{C}_{1}(i)\right)^{2}+\left(C_{2}(i)-\hat{C}_{2}(i)\right)^{2}\right]
$$

is minimized with respect to the parameters $k_{1}, k_{2}, \alpha, \beta$ and $\sigma_{1}^{p, i}, i=1, \ldots, 8$, where $C_{1}(i)$ and $C_{2}(i)$ are, respectively, the concentrations of cobalt and cadmium ions at the time points $i=1, \ldots, 8$ calculated from the solution of the system of time delayed differential equations corresponding to the choice of parameters $k_{1}, k_{2}, \alpha, \beta$ and $\sigma_{1}^{p, i}$, $i=1, \ldots, 8$.

Remark 1. The states before zero time point are obtained through interpolation of the measured data at the measurement time points prior to the zero time point. The cubic basis spline interpolation method (see [25]) is adopted to construct the fitting curve which passes through all the measurement data.

To use a gradient-based optimization technique, such as the SQP approximation scheme with active set strategy (see [24]), we need the gradient formulae of the cost function (4.2) with respect to the parameters $k_{1}, k_{2}, \alpha, \beta$ and $\sigma_{1}^{p, i}, i=1, \ldots, 8$. By virtue of Theorem 1, they are given by the following formulae

$$
\begin{aligned}
& \frac{\partial J_{1}}{\partial k_{1}}=-\frac{1}{V} \sum_{i=1}^{8} \int_{i-1}^{i} \sigma_{1}^{p, i} \lambda_{1}(t) C_{1}(t-2) \mathrm{d} t \\
& \frac{\partial J_{1}}{\partial k_{2}}=-\frac{1}{V} \sum_{i=1}^{8} \int_{i-1}^{i}\left(\Gamma-\sigma_{1}^{p, i}\right) \lambda_{2}(t) C_{2}(t-2) \mathrm{d} t, \\
& \frac{\partial J_{1}}{\partial \alpha}=\frac{1}{V} \sum_{i=1}^{8} \int_{i-1}^{i} \lambda_{1}(t) C_{2}(t-2) \mathrm{d} t, \\
& \frac{\partial J_{1}}{\partial \beta}=\frac{1}{V} \sum_{i=1}^{8} \int_{i-1}^{i} \lambda_{2}(t) C_{1}(t-2) \mathrm{d} t, \\
& \frac{\partial J_{1}}{\partial \sigma_{1}^{p, i}}=-\frac{1}{V} \int_{i-1}^{i}\left(k_{1} \lambda_{1}(t) C_{1}(t-2)-k_{2} \lambda_{2}(t) C_{2}(t-2)\right) \mathrm{d} t,
\end{aligned}
$$


where $\left(\lambda_{1}, \lambda_{2}\right)$ is the solution of the co-state system given below

$$
\begin{aligned}
& \frac{\mathrm{d} \lambda_{1}(t)}{\mathrm{d} t}=\left(\frac{Q}{V}+\frac{k_{1} \sigma_{1}^{p, i}}{V}\right) \lambda_{1}(t+2) e(6-t)-\frac{\beta}{V} \lambda_{2}(t+2) e(6-t), \\
& \frac{\mathrm{d} \lambda_{2}(t)}{\mathrm{d} t}=\left(\frac{Q}{V}+\frac{k_{2}\left(\Gamma-\sigma_{1}^{p, i}\right)}{V}\right) \lambda_{2}(t+2) e(6-t)-\frac{\alpha}{V} \lambda_{1}(t+2) e(6-t),
\end{aligned}
$$

where $t \in(i-1, i), i=1, \ldots, 8$, with the jump conditions

$$
\begin{aligned}
& \lambda_{1}\left(i^{+}\right)-\lambda_{1}\left(i^{-}\right)=-2\left(C_{1}(i)-\hat{C}_{1}(i)\right), \quad \text { for } \quad i=1, \ldots, 7, \\
& \lambda_{2}\left(i^{+}\right)-\lambda_{2}\left(i^{-}\right)=-2\left(C_{2}(i)-\hat{C}_{2}(i)\right), \quad \text { for } \quad i=1, \ldots, 7,
\end{aligned}
$$

and the terminal condition

$$
\begin{aligned}
& \lambda_{1}(8)=2\left(C_{1}(8)-\hat{C}_{1}(8)\right), \\
& \lambda_{2}(8)=2\left(C_{2}(8)-\hat{C}_{2}(8)\right), \\
& \lambda_{1}(t)=0, \quad \lambda_{2}(t)=0, \quad t>8 .
\end{aligned}
$$

Now, for each given parameters $k_{1}, k_{2}, \alpha, \beta$ and $\sigma_{1}^{p, i}, i=1, \ldots, 8$, the value of the cost function and the corresponding gradients with respect to these parameters can be calculated as detailed in the following algorithm.

Algorithm 1. Step 1. For each given parameters $k_{1}, k_{2}, \alpha, \beta$ and $\sigma_{1}^{p, i}, i=1, \ldots, 8$, compute the solution $\left(C_{1}, C_{2}\right)$ of the state of the dynamical system by solving the differential equations (2.2) forward from $t=0$ to $t=1$, where the initial conditions, i.e., $C_{1}(t)$ and $C_{2}(t)$ for $t \in[-2,0]$ constructed as indicated in Remark 1 . Then, the differential equations (2.2) are solved forward from $t=1$ to $t=2$, where the initial conditions, i.e., $C_{1}(t)$ and $C_{2}(t)$ for $t \in[0,1]$, constructed through interpolation by using linear function on the subinterval $[0,1]$. This process is continued until we obtain all values of $C_{1}(t)$ and $C_{2}(t)$ on the time interval [0,8].

Step 2. With the solution $\left(C_{1}, C_{2}\right)$ obtained in Step 1, the value of the cost function $J_{1}$ can be easily calculated by using (4.2).

Step 3. Solve the co-state system (4.4) with the terminal condition (4.6) backward from $t=8$ to $t=7$. Use the jump condition (4.5) to determine $\lambda_{1}\left(7^{-}\right), \lambda_{2}\left(7^{-}\right)$. Then, the co-state system (4.4) is solved backward from $t=7$ to $t=6$, where the terminal conditions $\lambda_{1}\left(7^{-}\right), \lambda_{2}\left(7^{-}\right)$and $\lambda_{1}(t), \lambda_{2}(t)$ for $t \in(7,8]$, constructed through interpolation by using linear function on subinterval $(7,8]$. This process is continued until we obtain all values of $\lambda_{1}(t)$ and $\lambda_{2}(t)$ on the time interval $[0,8]$.

Step 4. Compute the gradients of the cost function with respect to the system parameters $k_{1}, k_{2}, \alpha, \beta$ and the control parameters $\sigma_{1}^{p, i}, i=1, \ldots, 8$ according to (4.3).

With Algorithm 1, a gradient-based optimization technique, such as the SQP approximation scheme, can be used to minimize the cost function (4.2) with respect to the system parameters and the control parameters. The optimal parameters obtained 
are:

$k_{1}=7.828 \times 10^{-4}, k_{2}=2.823 \times 10^{-4}, \alpha=16.67, \beta=7.107 \times 10^{2}, \sigma_{1}^{p, 1}=6.5386 \times 10^{4}$, $\sigma_{1}^{p, 2}=1.0627 \times 10^{5}, \sigma_{1}^{p, 3}=8.9681 \times 10^{4}, \sigma_{1}^{p, 4}=1.11603 \times 10^{5}, \sigma_{1}^{p, 5}=1.13674 \times 10^{5}$, $\sigma_{1}^{p, 6}=9.835 \times 10^{4}, \sigma_{1}^{p, 7}=8.9673 \times 10^{4}, \sigma_{1}^{p, 8}=1.01823 \times 10^{5}$.

Let $\bar{C}_{j}, j=1,2$, be the concentrations of cobalt and cadmium ions corresponding to these optimal parameters. They are shown as dashed lines in Figures 1-2. After these optimal parameters are obtained, we move on to find the controls $U_{1}^{p}$ and $U_{2}^{p}$ in the next step. As we know, the zinc powder reaction surface areas used are rather excessive than required. We thus move to minimize the zinc powder reaction surface areas subject to the condition that the possible deviations of $C_{j}(t), j=1,2$, which are the solutions of the system of time delayed differential equations corresponding to the choice of the controls $U_{1}$ and $U_{2}$, from $\bar{C}_{j}(t), j=1,2$, are within an acceptable limit.

This can be stated as a time delayed optimal control problem in the form of Problem (P2), where

$$
J_{2}=\left(C_{1}(8)-\bar{C}_{1}(8)\right)^{2}+\left(C_{2}(8)-\bar{C}_{2}(8)\right)^{2}+\int_{0}^{8}\left(U_{1}^{p}(t)^{2}+U_{2}^{p}(t)^{2}\right) \mathrm{d} t
$$

is minimized with respect to the control parameters $\sigma_{1}^{p, i}$ and $\sigma_{2}^{p, i}, i=1, \ldots, 8$, subject to the continuous state inequality constraints

$$
C_{j, e}=e-\left(C_{j}(t)-\bar{C}_{j}(t)\right)^{2} \geq 0, \quad t \in[0,8], \quad j=1,2,
$$

where $e>0$ is an error bound.

Using the constraint transcription method (see (3.9)), each of the continuous state inequality constraints (4.8) is approximated by a sequence of inequality constraint in canonical form given below

$$
C_{j, \varepsilon}=\gamma+\int_{0}^{8} \mathcal{L}_{j, \varepsilon}\left(t, C_{j}(t)\right) \mathrm{d} t \geq 0, \quad j=1,2,
$$

where

$$
\mathcal{L}_{j, \varepsilon}\left(t, C_{j}(t)\right)= \begin{cases}e-\left(C_{j}(t)-\bar{C}_{j}(t)\right)^{2}, & \text { if } C_{j, e}<-\varepsilon \\ -\left(e-\left(C_{j}(t)-\bar{C}_{j}(t)\right)^{2}-\varepsilon\right)^{2} / 4 \varepsilon, & \text { if }-\varepsilon \leq C_{j, e} \leq \varepsilon \\ 0, & \text { if } C_{j, e}>\varepsilon\end{cases}
$$

Here, $\varepsilon>0$ is an adjustable parameter controlling the accuracy of the approximation, while $\gamma>0$ is an adjustable parameter controlling the feasibility of the constraints.

By virtue of Theorem 1, the gradient formulae of the cost function (4.7) with respect to the control parameters $\sigma_{1}^{p, i}$ and $\sigma_{2}^{p, i}, i=1, \ldots, 8$, in each time interval are given by

$$
\frac{\partial J_{2}}{\partial \sigma_{1}^{p, i}}=\int_{i-1}^{i}\left(2 \sigma_{1}^{p, i}-\frac{k_{1}}{V} \lambda_{0,1}(t) C_{1}(t-2)\right) \mathrm{d} t
$$




$$
\frac{\partial J_{2}}{\partial \sigma_{2}^{p, i}}=\int_{i-1}^{i}\left(2 \sigma_{2}^{p, i}-\frac{k_{2}}{V} \lambda_{0,2}(t) C_{2}(t-2)\right) \mathrm{d} t .
$$

where $\left[\lambda_{0,1}, \lambda_{0,2}\right]^{\top}$ is the solution of the co-state system given below

$$
\begin{aligned}
& \frac{\mathrm{d} \lambda_{0,1}(t)}{\mathrm{d} t}=\left(\frac{Q}{V}+\frac{k_{1} \sigma_{1}^{p, i}}{V}\right) \lambda_{0,1}(t+2) e(6-t)-\frac{\beta}{V} \lambda_{0,2}(t+2) e(6-t), \\
& \frac{\mathrm{d} \lambda_{0,2}(t)}{\mathrm{d} t}=\left(\frac{Q}{V}+\frac{k_{2} \sigma_{2}^{p, i}}{V}\right) \lambda_{0,2}(t+2) e(6-t)-\frac{\alpha}{V} \lambda_{0,1}(t+2) e(6-t),
\end{aligned}
$$

with the terminal condition

$$
\begin{aligned}
& \lambda_{0,1}(8)=2\left(C_{1}(8)-\bar{C}_{1}(8)\right), \\
& \lambda_{0,2}(8)=2\left(C_{2}(8)-\bar{C}_{2}(8)\right), \\
& \lambda_{0,1}(t)=0, \quad \lambda_{0,2}(t)=0, \quad t>8 .
\end{aligned}
$$

Meanwhile, the gradient formulae of the constraint functions (4.9) with respect to the control parameters $\sigma_{1}^{p, i}$ and $\sigma_{2}^{p, i}, i=1, \ldots, 8$, in each time interval are given by

$$
\begin{aligned}
& \frac{\partial C_{1, \varepsilon}}{\partial \sigma_{1}^{p, i}}=-\int_{i-1}^{i} \frac{k_{1}}{V} \lambda_{1,1}(t) C_{1}(t-2) \mathrm{d} t \\
& \frac{\partial C_{1, \varepsilon}}{\partial \sigma_{2}^{p, i}}=-\int_{i-1}^{i} \frac{k_{2}}{V} \lambda_{1,2}(t) C_{2}(t-2) \mathrm{d} t \\
& \frac{\partial C_{2, \varepsilon}}{\partial \sigma_{1}^{p, i}}=-\int_{i-1}^{i} \frac{k_{1}}{V} \lambda_{2,1}(t) C_{1}(t-2) \mathrm{d} t \\
& \frac{\partial C_{2, \varepsilon}}{\partial \sigma_{2}^{p, i}}=-\int_{i-1}^{i} \frac{k_{2}}{V} \lambda_{2,2}(t) C_{2}(t-2) \mathrm{d} t .
\end{aligned}
$$

where $\left[\lambda_{1,1}, \lambda_{1,2}, \lambda_{2,1}, \lambda_{2,2}\right]^{\top}$ is the solution of the co-state system given below

$$
\begin{aligned}
& \frac{\mathrm{d} \lambda_{1,1}(t)}{\mathrm{d} t}=-\frac{\partial \mathcal{L}_{1, \varepsilon}}{\partial C_{1}}+\left(\frac{Q}{V}+\frac{k_{1} \sigma_{1}^{p, i}}{V}\right) \lambda_{1,1}(t+2) e(6-t)-\frac{\beta}{V} \lambda_{1,2}(t+2) e(6-t), \\
& \frac{\mathrm{d} \lambda_{1,2}(t)}{\mathrm{d} t}=\left(\frac{Q}{V}+\frac{k_{2} \sigma_{2}^{p, i}}{V}\right) \lambda_{1,2}(t+2) e(6-t)-\frac{\alpha}{V} \lambda_{1,1}(t+2) e(6-t), \\
& \frac{\mathrm{d} \lambda_{2,1}(t)}{\mathrm{d} t}=\left(\frac{Q}{V}+\frac{k_{1} \sigma_{1}^{p, i}}{V}\right) \lambda_{2,1}(t+2) e(6-t)-\frac{\beta}{V} \lambda_{2,2}(t+2) e(6-t), \\
& \frac{\mathrm{d} \lambda_{2,2}(t)}{\mathrm{d} t}=-\frac{\partial \mathcal{L}_{2, \varepsilon}}{\partial C_{2}}+\left(\frac{Q}{V}+\frac{k_{2} \sigma_{2}^{p, i}}{V}\right) \lambda_{2,2}(t+2) e(6-t)-\frac{\alpha}{V} \lambda_{2,1}(t+2) e(6-t),
\end{aligned}
$$


with the terminal condition

$$
\begin{aligned}
& \lambda_{1,1}(8)=0, \lambda_{1,2}(8)=0, \\
& \lambda_{2,1}(8)=0, \lambda_{2,2}(8)=0, \\
& \lambda_{1,1}(t)=0, \lambda_{1,2}(t)=0, \quad t>8, \\
& \lambda_{2,1}(t)=0, \lambda_{2,2}(t)=0, \quad t>8,
\end{aligned}
$$

where

$$
\frac{\partial \mathcal{L}_{j, \varepsilon}}{\partial C_{j}}= \begin{cases}-2\left(C_{j}(t)-\bar{C}_{j}(t)\right), & \text { if } C_{j, e}<-\varepsilon, \\ \left(e-\left(C_{j}(t)-\bar{C}_{j}(t)\right)^{2}-\varepsilon\right)\left(C_{j}(t)-\bar{C}_{j}(t)\right) / \varepsilon, & \text { if }-\varepsilon \leq C_{j, e} \leq \varepsilon, \quad j=1,2 \\ 0, & \text { if } C_{j, e}>\varepsilon\end{cases}
$$

The way of adjusting $\varepsilon$ and $\gamma$ is detailed in the following algorithm.

Algorithm 2. Input parameters $\varepsilon, \gamma$.

Step 1. Choose $\sigma_{1}^{p, i, 0}$ and $\sigma_{2}^{p, i, 0}, i=1, \ldots, 8$. Solve the approximate Problem (P2) by using the SQP approximation scheme, where the values of the cost function and the corresponding gradients are calculated as detailed in Algorithm 1. Let the optimal solution obtained be denoted by $\sigma_{1}^{p, i, *}$ and $\sigma_{2}^{p, i, *}, i=1, \ldots, 8$.

Step 2. Check feasibility of $C_{j, e}\left(t, C_{j}\left(t \mid \sigma_{j}^{p, i, *}\right)\right) \geq 0, j=1,2, i=1, \ldots, 8$, for all $t \in[0,8]$. If $\sigma_{1}^{p, i, *}, \sigma_{2}^{p, i, *}, i=1, \ldots, 8$, are feasible, go to Step 4, otherwise, go to Step 3 .

Step 3. Set $\gamma=\gamma / 10$ and go to Step 1 .

Step 4. Set $\varepsilon=\varepsilon / 10$, if $\varepsilon>\varepsilon_{\min }$, then go to Step 1, else successfully exist.

The obtained optimal cobalt and cadmium ions concentrations are shown as solid lines in Figures 1-2. We see that these optimal concentrations remain on track to the measured data. Several measured data deviate rather far away from the calculated concentration. This is expected, as the measurements are carried out manually by using some special chemical instruments. Inevitably, it will lead to some measurement inaccuracy. However, the trajectory obtained does reflect the basic characteristic of the practical scenarios under study.

The obtained optimal control parameters are:

$\sigma_{1}^{p, 1}=1.08282 \times 10^{5}, \sigma_{1}^{p, 2}=1.57032 \times 10^{5}, \sigma_{1}^{p, 3}=1.23983 \times 10^{5}, \sigma_{1}^{p, 4}=1.55718 \times 10^{5}$, $\sigma_{1}^{p, 5}=1.58877 \times 10^{5}, \sigma_{1}^{p, 6}=1.43113 \times 10^{5}, \sigma_{1}^{p, 7}=1.24693 \times 10^{5}, \sigma_{1}^{p, 8}=1.24739 \times 10^{5}$, $\sigma_{2}^{p, 1}=5.20019 \times 10^{5}, \sigma_{2}^{p, 2}=4.6972 \times 10^{5}, \sigma_{2}^{p, 3}=4.97383 \times 10^{5}, \sigma_{2}^{p, 4}=4.60475 \times 10^{5}$, $\sigma_{2}^{p, 5}=4.52652 \times 10^{5}, \sigma_{2}^{p, 6}=4.63645 \times 10^{5}, \sigma_{2}^{p, 7}=4.73757 \times 10^{5}, \sigma_{2}^{p, 8}=4.61721 \times 10^{5}$.

As mentioned before, the amount of zinc powder actually added is expressed as a whole quantity and the reaction surface areas $U_{1}$ and $U_{2}$ can be converted to the weight according to the linear relationship between the weight and reaction surface area of zinc powder. Thus, the respective weight of zinc powder for cobalt and cadmium ions are shown in Figures 3-4. The total weight of zinc powder is shown in Figure 5. We see that the average weight is much lower than the one used in current practice, shown as dashed line. Our proposed method has found the optimal consumption of zinc powder 


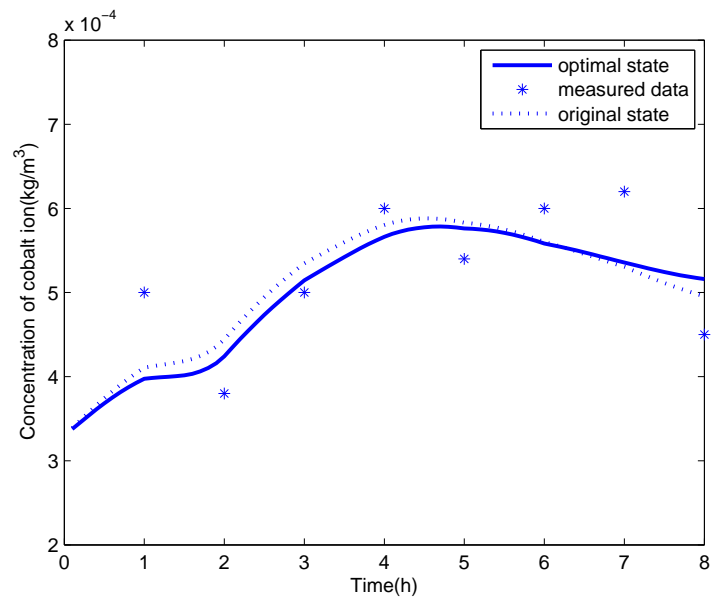

Figure 1: Cobalt ion concentration.

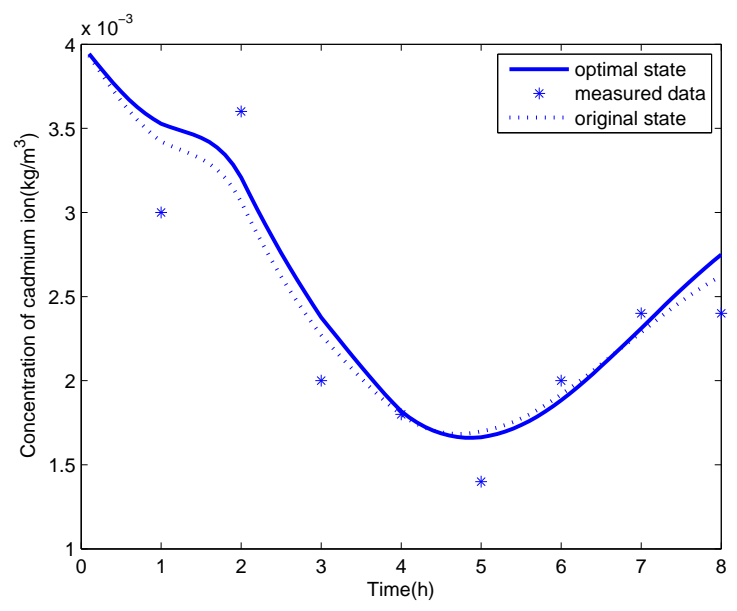

Figure 2: Cadmium ion concentration.

at each hour effectively.

\section{Conclusion}

Based on the metallic ion deposition mechanism and chemical kinetics, a dynamical model for the zinc sulphate electrolyte purification process was established. This model was described by a system of time delayed ordinary differential equations, in which some of the parameters are unknown. After the parameters have been identified, the problem of minimizing the consumption of zinc powder, which is used to remove the metallic ion impurities, can be formulated as an optimal control problem involving a time delayed dynamical system and subject to continuous state constraints. The control parametrization method, which involves approximating the control by a piecewise constant function, was applied to derive an approximate problem. This approximate problem can be solved using the SQP approximation method, which is an efficient gradient-based optimization method. The simulation results obtained have clearly indicated the effectiveness of the proposed method.

\section{Acknowledgment}

This work was supported by the Australian Research Council and National Natural Science Foundation of China under Grant No. 60634020 and 60874069. 

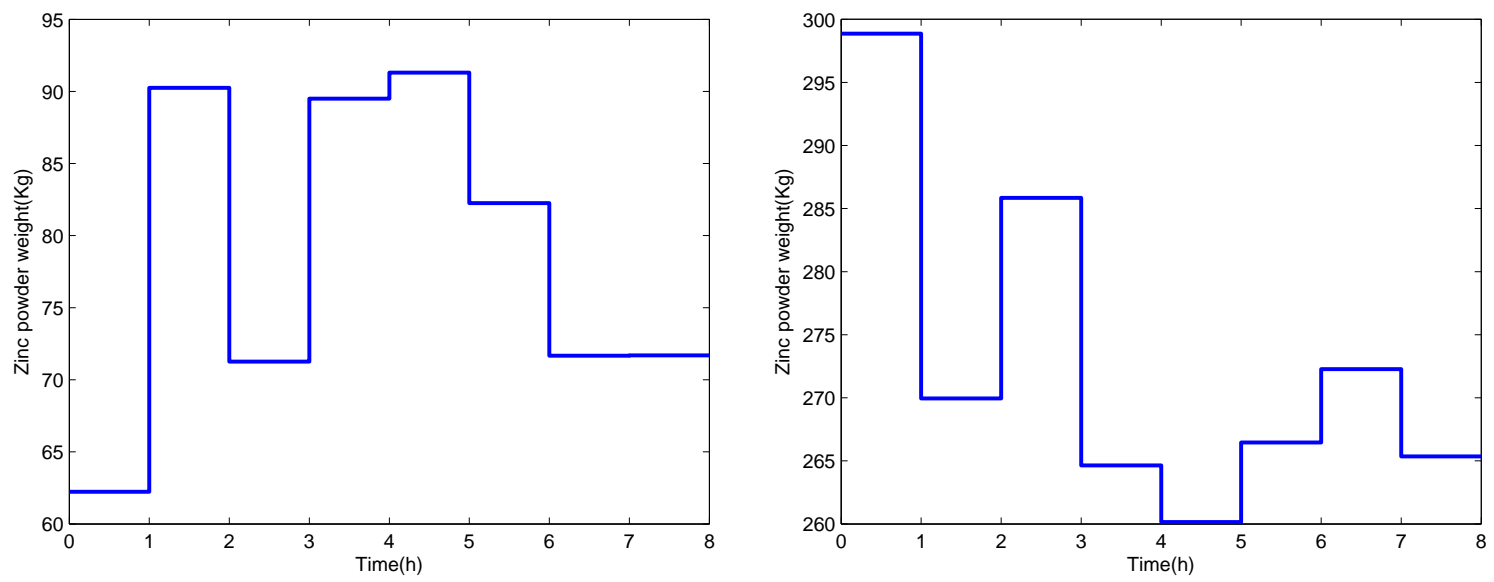

Figure 3: Zinc powder weight for cobalt ion. Figure 4: Zinc powder weight for cadmium ion.

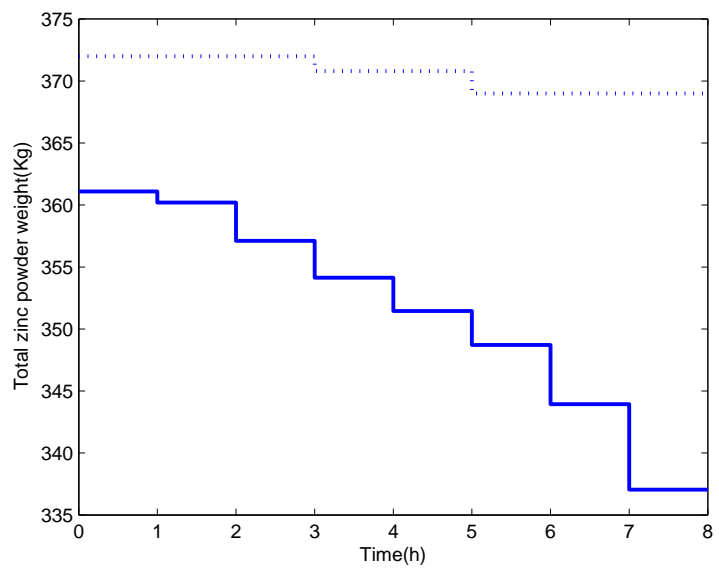

Figure 5: Total zinc powder weight. 


\section{References}

[1] G. C. Bratt, Impurity effect in the electrowinning of zinc and cadmium. Electrochemical Technology, 2(1964), 323-326

[2] D. R. Fosnacht, T. J. O'Keefe, The effects of certain impurities and their interactions on zinc electrowinning. Metallurgy Transactions, 14(1983), 645-655

[3] B. Nyman, A. Aaltonen, S. E. Hultholm, K. Karpale, Application of new hydrometallurgical developments in the Outokumpu HIKO process. Hydrometallurgy, 29(1992), 461-478

[4] V. Singh, Technological innovation in the zinc electrolyte purification process of a hydrometallurgical zinc plant through reduction in zinc dust consumption. Hydrometallurgy, 40(1996), 247-262

[5] R. Raghavan, P. K. Mohanan, S. K. Verma, Modified zinc sulphate solution purification technique to obtain low levels of cobalt for the zinc electrowinning process. Hydrometallurgy, 51(1999), 187-206

[6] J. Moghaddam, R. Sarraf-Mamoory, M. Abdollahy, Y. Yamini, Purification of zinc ammoniacal leaching solution by cementation: Determination of optimum process conditions with experimental design by Taguchi's method. Separation and Purification Technology, 51(2006), 157-164

[7] K. Tozawa, T. Nishimura, M. Akahori, A. Malaga, Comparison between purification processes for zinc leach solutions with arsenic and antimony trioxides. Hydrometallurgy, 30(1992), 445-461

[8] J. A. Sędzimir, Precipitation of metals by metals (cementation) - kinetics, equilibria. Hydrometallurgy, 64(2002), 161-167

[9] A. A. Taha, S. A. H. Abd El-Ghani, Effect of surfactants on the cementation of cadmium. Journal of Colloid and Interface Science, 280(2004), 9-17

[10] T. M. Dreher, A. Nelson, G. P. Demopoulos, D. Filippou, The kinetics of cobalt removal by cementation from an industrial zinc electrolyte in the presence of $\mathrm{Cu}$, Cd, Pb, Sb and Sn additives. Hydrometallurgy, 60(2001), 105-116

[11] V. Van Der Pas, D. B. Dreisinger, A fundamental study of cobalt cementation by zinc dust in the presence of copper and antimony additives. Hydrometallurgy, 43(1996), 187-205

[12] M. Karavasteva, The influence of copper on the effect of certain surfactants during the cementation of cadmium by suspended zinc particles. Hydrometallurgy, 48(1998), 361-366 
[13] J. Näsi, Statistical analysis of cobalt removal from zinc electrolyte using the arsenic-activated process. Hydrometallurgy, 73(2004), 123-132

[14] D. Yang, G. Xie, G. Zeng, J. Wang, R. Li, Mechanism of cobalt removal from zinc sulfate solutions in the presence of cadmium. Hydrometallurgy, 81(2006), 62-66

[15] K. Stole-Hansen, D. A. Wregget, D. Gowanlock, P. E. Thwaites, Model based analysis and control of a cementation process. Computers \& Chemical Engineering, 21(1997), S1099-S1103

[16] S. Salehi, M. Shahrokhi, Two observer-based nonlinear control approaches for temperature control of a class of continuous stirred tank reactors. Chemical Engineering Science, 63(2008), 396-403

[17] P. Menoud, L. Cavin, A. Renken, Modelling of heavy metals adsorption to a chelating resin in a fluidized bed reactor. Chemical Engineering and Processing, 37(1998), 89-101

[18] E. B. Lynch, W. F. Ramirez, Real-time time-optimal control of a stirred tank reactor using Kalman filtering for state estimation, AIChE Journal, 21(4)(1975), 799-804

[19] T. D. Knapp, H. M. Budman, G. Broderick, Adaptive control of a CSTR with neural work model, Journal of Process Control, 11(2001), 53-68

[20] W. A. Cebuhar, V. Costanza, Nonlinear control of CSTR's, Chemical Engineering Science, 39(12)(1984), 1715-1722

[21] R. B. Martin, K. L. Teo, Optimal control of drug administration in cancer chemotherapy. Singapore: World Scientific, 1994.

[22] L. S. Jennings, K. L. Teo, A computational algorithm for functional inequality constrained optimization problems. Automatica, 26(2)(1990), 371-375

[23] K. Kaji, K. H. Wong, Nonlinearly constrained time-delayed optimal control problems. Journal of Optimization Theory and Applications, 82(2)(1994), 295-313

[24] K. L. Teo, C. J. Goh, K. H. Wong. A Unified computational approach to optimal control problems. Longman Scientific and Technical, New York, 1991.

[25] V. Rehbock, K. L. Teo, L. S. Jennings, Suboptimal feedback control for a class of nonlinear systems using spline interpolation, Discrete and Continuous Dynamical Systems, 1(2)(1995), 223-236

[26] K. Schittkowski (2004). NLPQLP: A Fortran Implementation of a Sequential Quadratic Programming Algorithm with Distributed and Non-monotone Line Search. User's Guide Version 2.0, University of Bayreuth. 